\title{
Clear Sky Identification Using Data from Remote Sensing Systems at ARM's Southern Great Plains Site
}

\author{
L. Delle Monache, D. Rodriguez and R. Cederwall
}

\section{June 27, 2000}

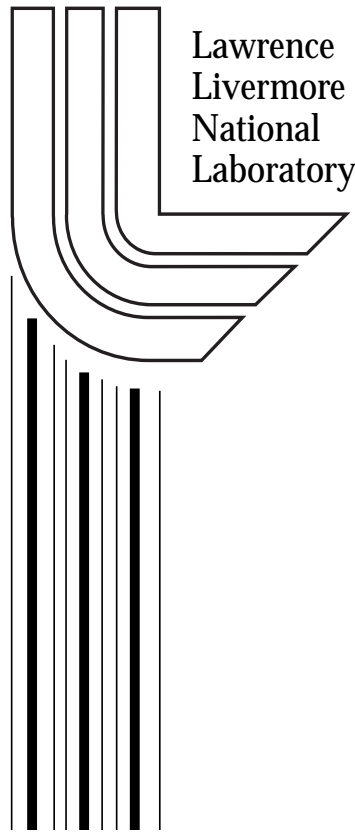




\section{DISCLAIMER}

This document was prepared as an account of work sponsored by an agency of the United States Government. Neither the United States Government nor the University of California nor any of their employees, makes any warranty, express or implied, or assumes any legal liability or responsibility for the accuracy, completeness, or usefulness of any information, apparatus, product, or process disclosed, or represents that its use would not infringe privately owned rights. Reference herein to any specific commercial product, process, or service by trade name, trademark, manufacturer, or otherwise, does not necessarily constitute or imply its endorsement, recommendation, or favoring by the United States Government or the University of California. The views and opinions of authors expressed herein do not necessarily state or reflect those of the United States Government or the University of California, and shall not be used for advertising or product endorsement purposes.

Work performed under the auspices of the U. S. Department of Energy by the University of California Lawrence Livermore National Laboratory under Contract W-7405-Eng-48.

This report has been reproduced directly from the best available copy.

Available to DOE and DOE contractors from the

Office of Scientific and Technical Information

P.O. Box 62, Oak Ridge, TN 37831

Prices available from (423) 576-8401

http://apollo.osti.gov/bridge/

Available to the public from the National Technical Information Service

U.S. Department of Commerce 5285 Port Royal Rd., Springfield, VA 22161 http://www.ntis.gov/

OR

Lawrence Livermore National Laboratory Technical Information Department's Digital Library http://www.llnl.gov/tid/Library.html 


\title{
Clear Sky Identification Using Data from Remote Sensing Systems at ARM's Southern Great Plains Site
}

\author{
Luca Delle Monache \\ Daniel Rodriguez \\ Richard Cederwall
}

\section{Introduction}

Clouds profoundly affect our weather and climate due, in large part, to their interactions with radiation. Unfortunately, our understanding of these interactions is, at best, incomplete, making it difficult to improve the treatment of atmospheric radiation in climate models. The improved treatment of clouds and radiation, and a better understanding of their interaction, in climate models is one of the Department of Energy's Atmospheric Radiation Measurement (ARM) Program's major goals. To learn more about the distribution of water and ice, i. e., clouds, within an atmospheric column, ARM has chosen to use the remote sensing of clouds, water vapor and aerosols at its three climatologically-diverse sites as its primary observational method. ARM's most heavily instrumented site, which has operated continuously for more than a decade, is its Southern Great Plains (SGP) Central Facility, located near Lamont, OK. Cloud-observing instruments at the Central Facility include the Whole Sky Imager, ceilometers, lidar, millimeter cloud radar, microwave radiometers and radiosondes.

The Whole-Sky Imager (WSI) is one of ARM's premiere instruments for cloud detection (Shields, et al., 1990). The WSI is a ground-based, electronic imaging system, designed for acquiring images over the sky's dome through spectral (red, blue and infrared) filters. This system, which passively measures microwave emissions emanating from the sky, operates continuously. It normally acquires images once every 10 minutes. From these images, the distribution of clouds overhead can be determined from observed radiances (540 X 540 pixel resolution) using an automated cloud decision algorithm and related processing. After calibrating the 16-bit data, the algorithm calculates the ratios between the red and blue radiances and their corresponding background, or "cloud- and aerosol-free", values on a pixel-by-pixel basis. Pixels are cloudy or clear, depending on whether the ratios are less than or greater than prescribed thresholds. In forming these ratios, the program has historically relied on a library of clear sky ratio images. While the assemblage cover the necessary range of solar elevation and azimuth angles comprehensively, the library is, unfortunately, site dependent; that is, the images at the SGP site, painstakingly collected from many year's worth of data, are unsuitable for use at ARM's Tropical West Pacific and North Slopes of Alaska sites.

To liberate the WSI algorithm from its need to use site-dependent data, the Santa Barbara DISORT Atmospheric Radiative Transfer (SBDART) code has been adopted to estimate background (red and blue) radiances at any point on the earth's surface 
(Ricchiazzi et al., 1998). SBDART is designed for analyzing a wide variety of radiative transfer problems encountered in satellite remote sensing and atmospheric energy budget studies. The program is based on a collection of highly developed and reliable physical models, which have been developed by the atmospheric science community over the past few decades. The discrete ordinate method provides a numerically stable algorithm for solving the equations of plane-parallel radiative transfer in a vertically inhomogeneous atmosphere. It is unclear, however, whether the assumption of planeparallel radiative transfer yields satisfactory results when computing clear-sky radiation values, especially in the case of low sun angles. The aim of this work is to devise a set of procedures for identifying cloud-free days at the SGP site. Ultimately, such identifications will allow SBDART outputs to be validated as a function of angular distance from zenith via comparisons with WSI measurements of "pristine", daytime skies.

\section{Identification of Clear Skies}

For our purposes an ideal, or pristine, day is one in which the sky is free of clouds, and the aerosol load and moisture content in the atmosphere are low. The latter two factors were not explicitly considered in our study, but their effects could have been evaluated from the available data. Our procedures for identifying cloud-free days are based on the visualization and comparison of data from two of ARM's instruments, effectively colocated at the Central Facility: the Microwave Water Radiometer (MWR) and the Micropulse Lidar (MPL). The zenith-pointing MWR provides column-integrated amounts of water vapor and liquid water, approximately once every 30 seconds, while the MPL, a ground-based, optical remote sensing system for determining the altitude of clouds overhead, provides averages of cloud base heights once every 60 seconds. MWR and MPL data collections from January and February, 1999, and from July and August, 1999, were examined because of an interest in finding seasonal examples of cloud-free days at a continental location.

Data visualization offers the following outcomes:

1. The skies are clear, i.e., the liquid water path (LWP) values are small and constant, and no cloud base exists below the pure ice-phase level.

Based on expert advice and personal experience, "small and constant" refers to hourly-averaged LWP values that are less than $0.03 \mathrm{~mm}$ (the retrieval noise threshold), and hourly-averaged standard deviations (STDs) that are less than $0.006 \mathrm{~mm}$. Note that the MPL data are checked to make sure no ice-phase clouds are present.

2. The clouds are homogeneous, both in terms of droplet size and layer thickness; the LWP values are large, but their hourly-averaged STDs are small.

3. The clouds are variable, leading to large LWPs and STDs.

Examples of two cloudy scenarios are given below. In the first figure, dated 01-27-1999, the measurements of LWP made by the MWR, where LOS refers to line-of-sight, are 
plotted over a 24-hour period. However, since we have an interest only in daytime measurements (more specifically, those times at which the sun is five degrees, or more, above the horizon), stippled areas, corresponding to nighttime hours, have been added for purposes of delineation. The base heights of the cloud, which are indicated by plus signs, can be estimated from the scale on the right-hand axis. The gray horizontal bar, which spans the daylight hours, represents our chosen threshold $(0.03 \mathrm{~mm})$ for the LWP. The red horizontal bar, covering the same time period, represents the average of the LWP measurements during the daytime.

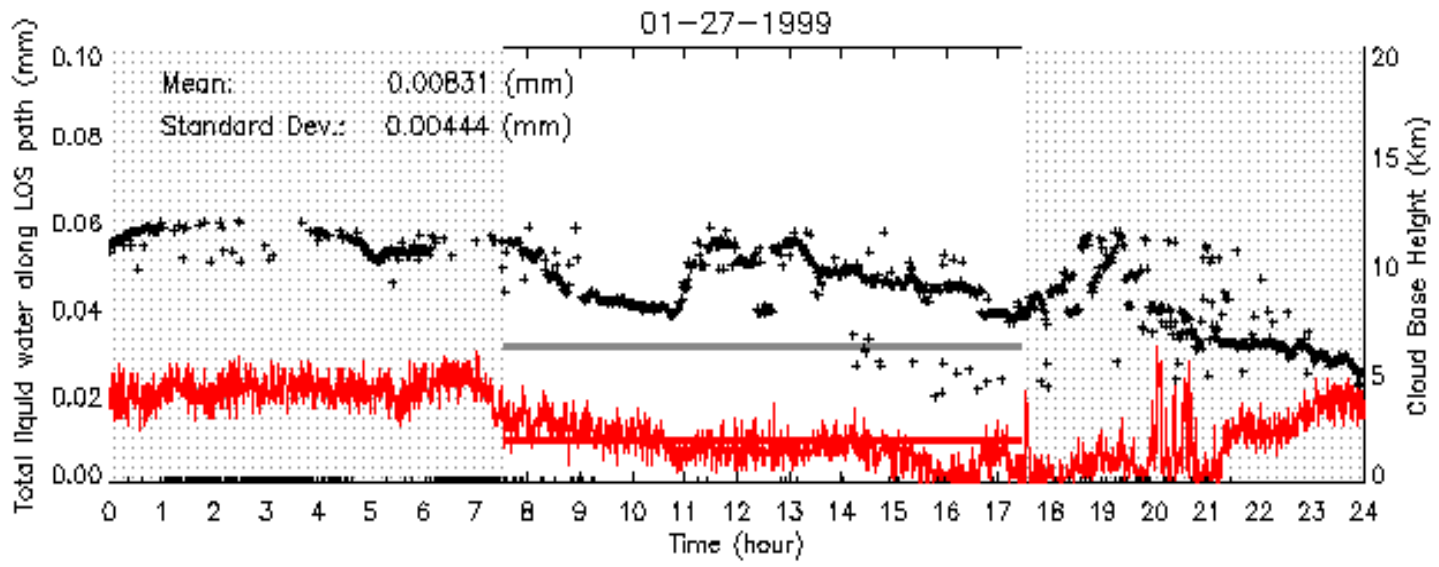

As can be seen, all LWP values fall below the $0.03 \mathrm{~mm}$ threshold... also indicated by the daytime mean and standard deviation of the MWR data in the upper left-hand corner of the plot...but they do so in the presence of a layer of cirrus clouds. This figure demonstrates the need to view both sets of data simultaneously, since the MWR is incapable of detecting ice crystals. The existence of a persistent cirrus deck means that the entire data set must be eliminated from consideration.

Like its predecessor the second figure, dated 02-21-1999, illustrates a cloud-filled sky, but one in which the clouds are composed of water, not ice.

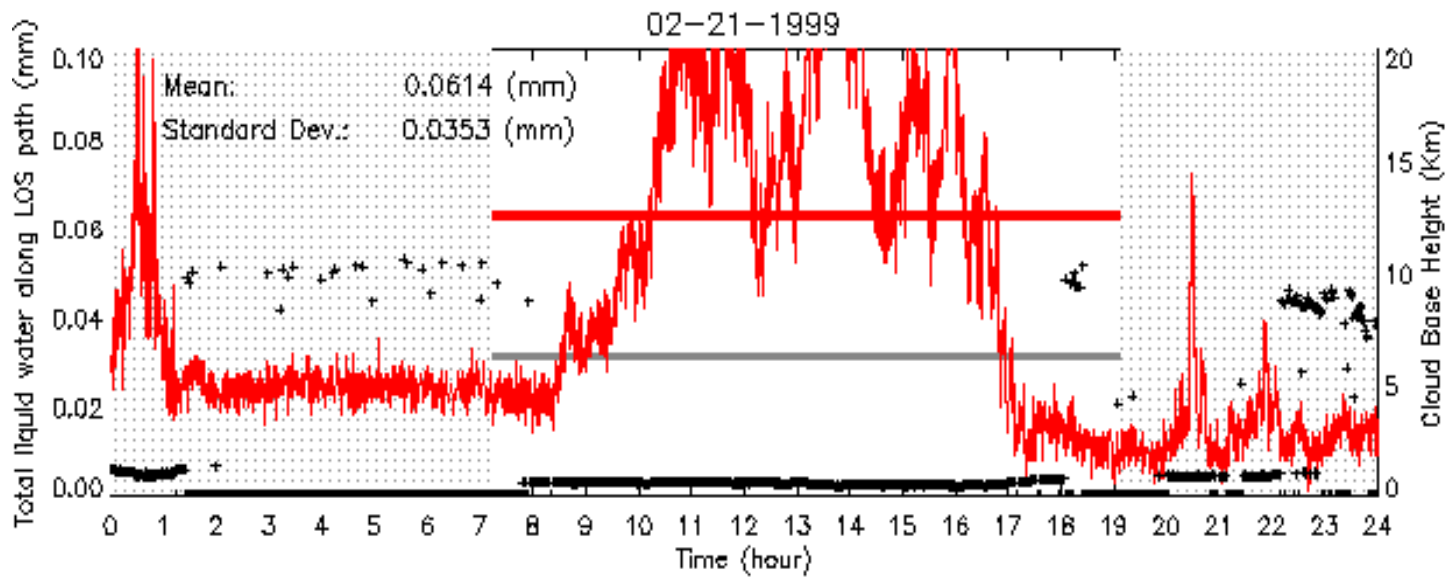

Although weather observations are missing on this particular day, the cloud-height data suggest that fog settled over the Central Facility during the night, followed by a slight lifting during the day as a result, perhaps, of surface heating. The increase in 
condensation, as indicated by a rapid increase in the LWP values, may be due in turn to atmospheric heating. In any event, the combined data suggest that the entire day is unsuitable for our purposes.

In the tables below, we identify the days of the month (January, February, July and August of 1999) that satisfy our selection criteria. When a day is labeled "clear" (in the second column labeled "MWR + MPL"), the MWR-measured LWP must be less than $0.03 \mathrm{~mm}$, its standard deviation must be less than $0.006 \mathrm{~mm}$, and clouds cannot be present overhead, as detected by MPL, for most of the daylight hours. Notes in the third column refer to human weather observations made at the Central Facility. Like the Whole Sky Imager's view of the world, these observations encompass the entire sky, as opposed to the narrow fields of view obtainable with the MWR and MPL, so they may be quite useful, when available.

Month: January

\begin{tabular}{|l|l|l|l|}
\hline Day & MWR+MPL & Observation & Pristine? \\
\hline 25 & Clear & Mostly Broken & No \\
\hline 27 & Clear & Clear, Cloudy and Broken & No \\
\hline
\end{tabular}

Month: February

\begin{tabular}{|l|l|l|l|}
\hline Day & MWR+MPL & Observation & Pristine? \\
\hline 3 & Clear & Clear form 7 to 11 a.m. & No \\
\hline 4 & Clear & OK & Yes! \\
\hline 12 & Clear & OK & Yes! \\
\hline 13 & Clear & Scattered & No \\
\hline 14 & Clear & No Data & No \\
\hline 15 & Clear & Clear from 11 a.m. to 2 p.m. & No \\
\hline 17 & Clear & Mostly Scattered & No \\
\hline 23 & Clear & Clear from 11 a.m. to 4 p.m. & No \\
\hline 24 & Clear & Clear from 7 a.m. to 1 p.m. & No \\
\hline 28 & Clear & OK & Yes! \\
\hline
\end{tabular}

Month: July

\begin{tabular}{|l|l|l|l|}
\hline Day & MWR+MPL & Observation & $\begin{array}{l}\text { Pristine } \\
?\end{array}$ \\
\hline 5 & Clear & OK from 11 a.m. to 2 p.m. & No \\
\hline 8 & Clear & $\begin{array}{l}\text { Scattered from } 3 \text { to } 5 \text { p.m. } \\
\text { and from } 8 \text { to } 9 \text { p.m. }\end{array}$ & No \\
\hline 9 & Clear & OK & Yes! \\
\hline 13 & Clear & Obscured from 8 to 9 a.m. & No \\
\hline 14 & Clear & No Data & No \\
\hline 15 & Clear & Partially Clear & No \\
\hline 17 & Clear & No Data & No \\
\hline
\end{tabular}




\begin{tabular}{|l|l|l|l|}
\hline 21 & Clear & Partially Scattered & No \\
\hline 22 & Clear & Mostly Scattered & No \\
\hline 23 & Clear & No data & No \\
\hline
\end{tabular}

Month: August

\begin{tabular}{|l|l|l|l|}
\hline Day & MWR+MPL & Observation & $\begin{array}{l}\text { Pristine } \\
?\end{array}$ \\
\hline 11 & Clear & Scattered & No \\
\hline 13 & Clear & Partially Scattered & No \\
\hline 14 & Clear & Partially Clear & No \\
\hline 15 & Clear & No Data & No \\
\hline 16 & Clear & Partially Scattered & No \\
\hline 17 & Clear & All clear but at 3 p.m. & Yes! \\
\hline 18 & Clear & All clear but at 3 p.m. & Yes! \\
\hline 19 & Clear & Mostly Scattered & No \\
\hline 20 & Clear & Mostly Scattered & No \\
\hline 22 & Clear & Scattered & No \\
\hline 23 & Clear & All clear but at 12 a.m. & Yes! \\
\hline 24 & Clear & Mostly Clear & Yes! \\
\hline 25 & Clear & OK & Yes! \\
\hline 29 & Clear & Scattered & No \\
\hline
\end{tabular}

Lastly, the next four plots---two wintertime and two summertime---illustrate either completely pristine days, or parts of pristine days. The tables below each plot provide hourly means and standard deviations of the measured liquid water paths in millimeters. Negative means are indications of retrieval uncertainty, resulting from regressing to a mean, and are within the noise limits of the MWR.

Winter pristine days: 


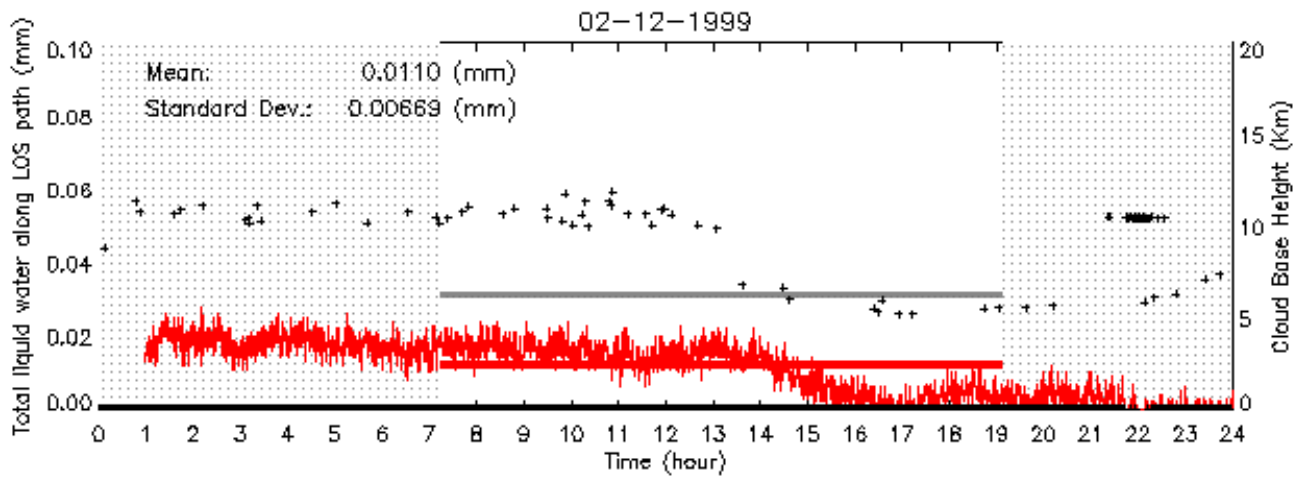

\begin{tabular}{|ccccccccc|}
\hline Hour & Mean & St. Dev. & Hour & Mean & St. Dev. & Hour & Mean & St. Dev. \\
1 & 0.013 & 0.0 & 9 & 0.016 & 0.0029 & 17 & 0.0017 & 0.0027 \\
2 & 0.018 & 0.0034 & 10 & 0.017 & 0.0027 & 18 & 0.0018 & 0.0044 \\
3 & 0.018 & 0.0032 & 11 & 0.015 & 0.0027 & 19 & 0.0044 & 0.0030 \\
4 & 0.018 & 0.0028 & 12 & 0.013 & 0.0031 & 20 & 0.0028 & 0.0029 \\
5 & 0.018 & 0.0027 & 13 & 0.015 & 0.0029 & 21 & 0.0032 & 0.0028 \\
6 & 0.017 & 0.0027 & 14 & 0.015 & 0.0026 & 22 & 0.0017 & 0.0029 \\
7 & 0.015 & 0.0029 & 15 & 0.010 & 0.0035 & 23 & -0.0014 & 0.0026 \\
8 & 0.016 & 0.0028 & 16 & 0.0049 & 0.0029 & 24 & -0.0015 & 0.0027 \\
\hline
\end{tabular}

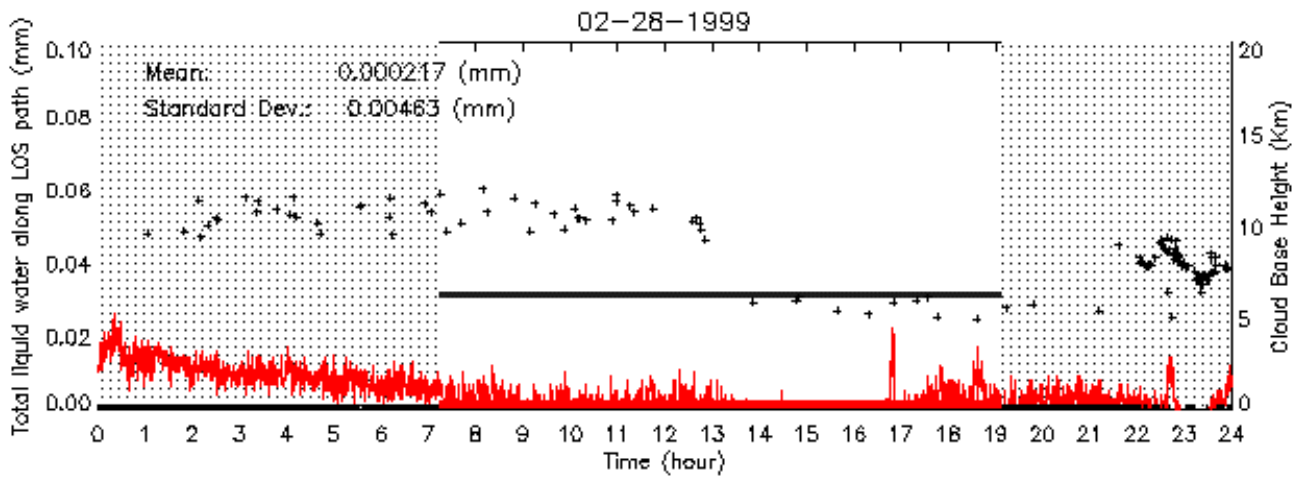

\begin{tabular}{|clcclclll|}
\hline Hour & Mean & St. Dev. & Hour & Mean & St. Dev. & Hour & Mean & St. Dev. \\
1 & 0.015 & 0.0040 & 9 & 0.0026 & 0.0031 & 17 & -0.0012 & 0.0059 \\
2 & 0.012 & 0.0027 & 10 & 0.00086 & 0.0031 & 18 & 0.0019 & 0.0038 \\
3 & 0.010 & 0.0024 & 11 & 0.00077 & 0.0026 & 19 & 0.0040 & 0.0040 \\
4 & 0.0097 & 0.0024 & 12 & 0.0015 & 0.0027 & 20 & 0.0019 & 0.0030 \\
5 & 0.0089 & 0.0027 & 13 & 0.0022 & 0.0027 & 21 & 0.0028 & 0.0027 \\
6 & 0.0070 & 0.0025 & 14 & -0.0013 & 0.0031 & 22 & 0.0017 & 0.0026 \\
7 & 0.0062 & 0.0026 & 15 & -0.0048 & 0.0030 & 23 & -1.4 & 12. \\
8 & 0.0035 & 0.0027 & 16 & -0.0066 & 0.0028 & 24 & -0.033 & 0.068 \\
\hline
\end{tabular}

Summer pristine days: 


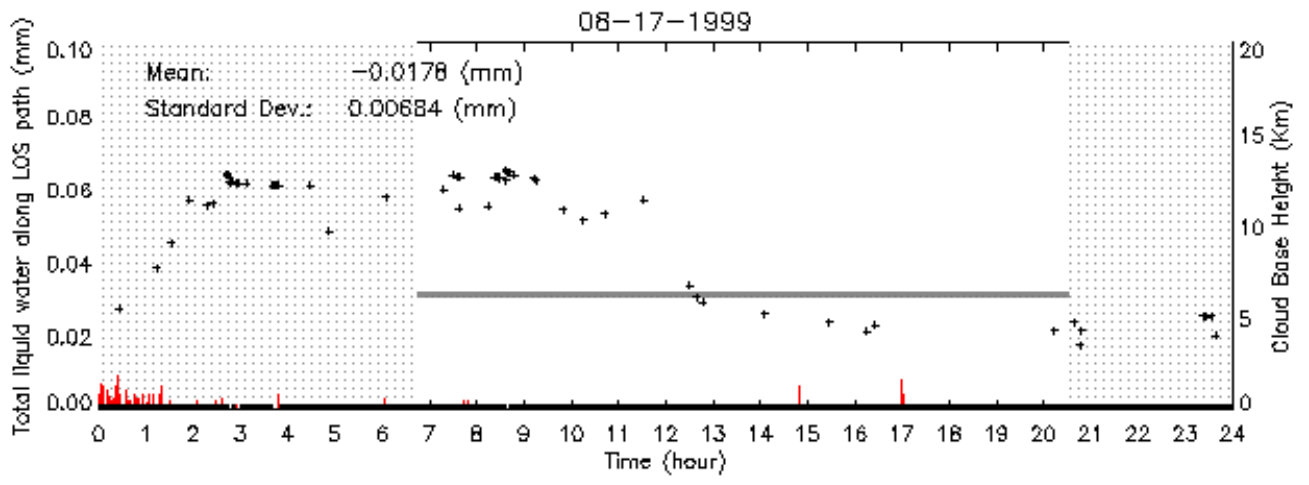

\begin{tabular}{|c|c|c|c|c|c|c|c|c|}
\hline Hour & Mean & St. Døv. & Hour & Mean & St. Dev. & Hour & Mean & St. Dev. \\
\hline 1 & -0.0015 & 0.0038 & 9 & -0.011 & 0.0039 & 17 & -0.018 & 0.0067 \\
\hline 2 & -0.0040 & 0.0036 & 10 & -0.013 & 0.0035 & 18 & -0.015 & 0.0055 \\
\hline 3 & -0.0053 & 0.0038 & 11 & -0.014 & 0.0034 & 19 & -0.021 & 0.0040 \\
\hline 4 & -0.0068 & 0.0034 & 12 & -0.015 & 0.0034 & 20 & -0.020 & 0.0044 \\
\hline 5 & -0.0090 & 0.0031 & 13 & -0.021 & 0.0037 & 21 & -0.024 & 0.0038 \\
\hline 6 & -0.0080 & 0.0033 & 14 & -0.022 & 0.0034 & 22 & -0.022 & 0.0037 \\
\hline 7 & -0.0068 & 0.0037 & 15 & -0.023 & 0.0060 & 23 & -0.022 & 0.0040 \\
\hline 8 & -0.0082 & 0.0041 & 16 & -0.025 & 0.0038 & 24 & -0.023 & 0.0030 \\
\hline
\end{tabular}

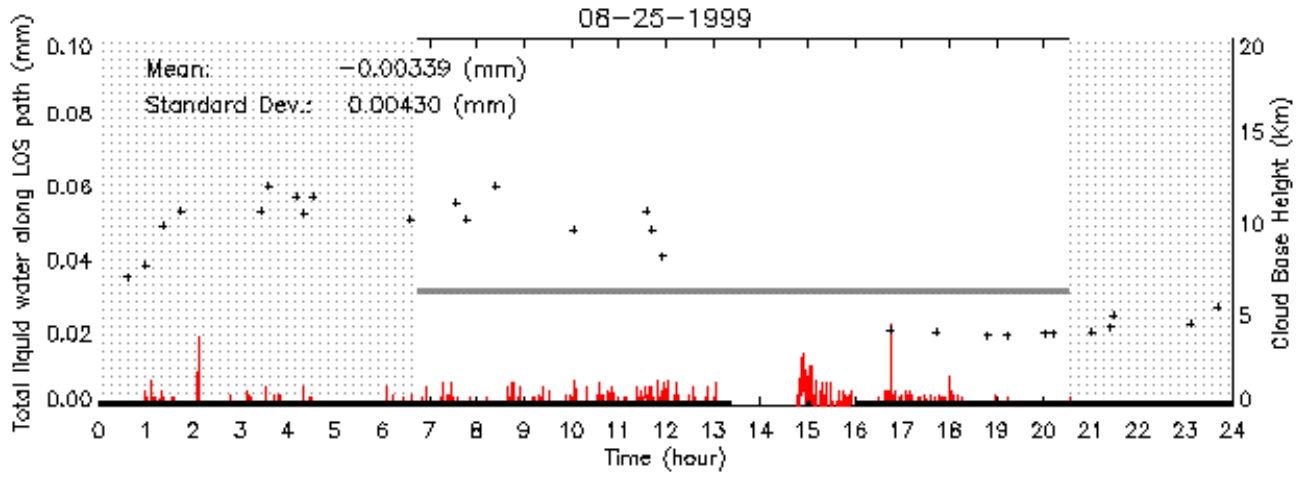

\begin{tabular}{|clccccccc|}
\hline Hour & Mean & St. Dev. & Hour & Mean & St. Dev. & Hour & Mean & St. Dev. \\
1 & -0.010 & 0.0046 & 9 & -0.0036 & 0.0041 & 17 & -0.0027 & 0.0041 \\
2 & -0.0034 & 0.0036 & 10 & -0.0030 & 0.0032 & 18 & -0.0030 & 0.0041 \\
3 & -0.0053 & 0.0039 & 11 & -0.0030 & 0.0035 & 19 & -0.0044 & 0.0036 \\
4 & -0.0046 & 0.0037 & 12 & -0.0015 & 0.0034 & 20 & -0.0065 & 0.0037 \\
5 & -0.0059 & 0.0033 & 13 & -0.0026 & 0.0035 & 21 & -0.0093 & 0.0032 \\
6 & -0.0060 & 0.0035 & 14 & -0.0053 & 0.0035 & 22 & -0.010 & 0.0037 \\
7 & -0.0049 & 0.0036 & 15 & 0.0038 & 0.0044 & 23 & -0.014 & 0.0038 \\
8 & -0.0031 & 0.0034 & 16 & -0.0012 & 0.0044 & 24 & -0.012 & 0.0036 \\
\hline
\end{tabular}

\section{Concluding Remarks}


Recall that the identification of pristine days at ARM's Southern Great Plains site was one of several steps leading to our ultimate goal: the determination of whether SBDART, as a tool, was effective for estimating the red and blue background radiances at ANY site around the globe. Were we to proceed, our next step in this process would be to compare the radiances from the Whole Sky Imager on the days identified as being cloud-free against the corresponding estimates from SBDART. In all likelihood, the level of agreement between the two would diminish outwards from the zenith towards the horizon, regardless of season or time of day. A determination would have to be made as to whether the falloff in accuracy was within acceptable limits. If not, would the coding associated with a plane-parallel assumption have to be replaced with coding that portrayed the atmosphere as an arching layer? Furthermore, to establish once and for all that SBDART was, indeed, a site-independent tool, the foregoing analyses would have to be repeated at ARM's Tropical West Pacific and North Slope of Alaska sites.

\section{References}

U.S. Department of Energy (DOE), 1996: Science Plan for the Atmospheric Radiation Measurement Program (ARM), DOE/ER-0670T, U.S. Department of Energy, Washington, D.C.

J.E. Shields, T.L. Koehler, M.E. Karr. \& R.W. Johnson, 1990: Automated Cloud Cover and Visibility Systems for Real Time Applications. University of California, San Diego, Scripps Institution of Oceanography, Marine Physical Laboratory, Optical Systems Group Technical Note No. 217.

P. Ricchiazzi, S. Yang, C. Gautier \& D. Sowle, 1998. SBDART: A Research and Teaching Software Tool for Plane-Parallel Radiative Transfer in the Earth's Atmosphere. Bullettin of the Amer. Meteor. Soc., Vol 79 No. 10.

\section{Appendix}

Program list:

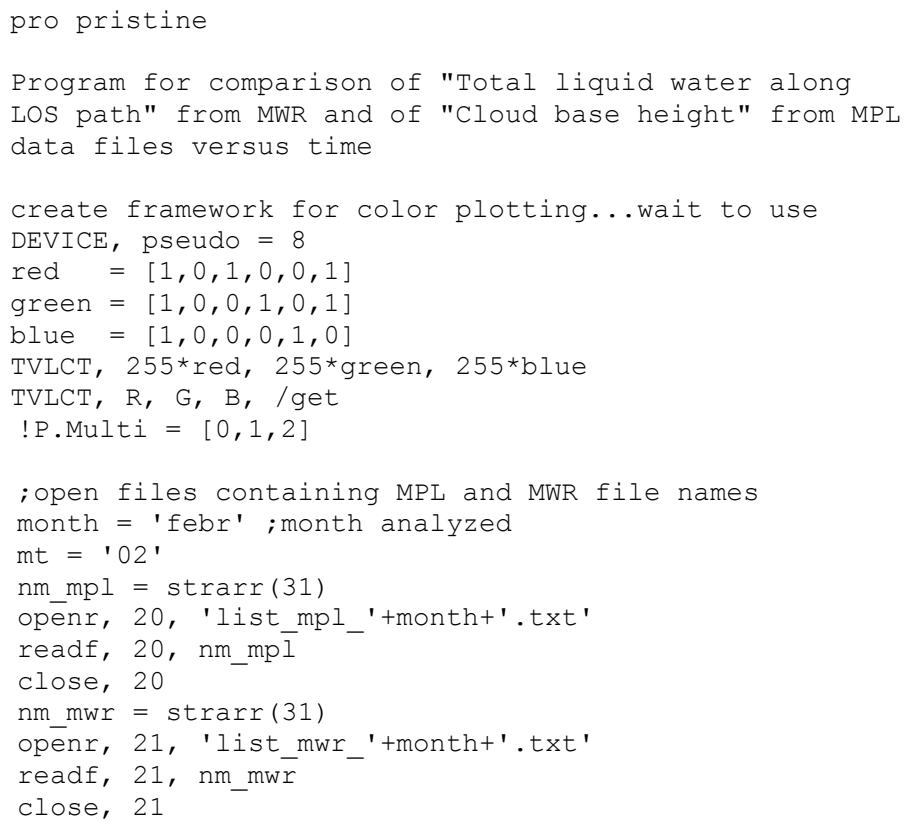


for iii $=21,31$ do begin ; Main loop over the days of the month

; setting of the file name for the current day

if iii lt 10 then begin

day $=\operatorname{string}($ format $='($ II)', iii)

day $=$ ' 0'+day

endif else begin

day $=\operatorname{string}($ format $='($ I2)', iii)

endelse

; check if for the current day both the MWR and MPL files are available str_mwr = 'sgpmwrlosc1.b1.1999'+mt+day

flag mwr $=1$

ind $\bar{m} w r=0$

while (ind mwr le 30) and (flag mwr eq 1) do begin

res_mwr $=\operatorname{strpos}\left(n m \_m w r\left(i \bar{n} d \_m w r\right)\right.$, str_mwr)

if $\bar{r}$ es mwr ne -1 thēn begin

$\mathrm{flag} \operatorname{mwr}=0$

endif else begin

ind $m w r=$ ind $m w r+1$

end endelse

; in the winter month the mpl file name strings are longer

; by four character ("nor1")

if ( $m$ t ne 01) and (mt ne 02)) then begin

st $=$ ' '

endif else begin

st $=$ 'nor 1 '

endelse

str_mpl = 'sgpmpl'+st+'campc1.c1.1999'+mt+day

flag $\mathrm{mpl}=1$

ind_mpl $=0$

while (ind mpl le 30) and (flag mpl eq 1) do begin

res_mpl $=\operatorname{strpos}\left(n m \_m p l\left(i \bar{n} d \_m p l\right)\right.$, str_mpl)

if res mpl ne -1 thēn begin

flag mpl $=0$

endif else begin

ind_mpl $=$ ind $m p l+1$

endelsēe

end

flag_sum = flag_mwrtflag_mpl

; if one file is not available this day is skipped

if (flag_sum gt 0 ) then begin

goto, JUMP

endif

; Open and read NetCDF files

; --- storage of the year, month and day relative to MWR data

name $\operatorname{mwr}=' \ldots /$ input $/ \operatorname{mwr} /$ '+month+'/'+nm mwr (ind mwr)

year $=$ strmid (name_mwr, 33,4)

$\mathrm{mm}=$ strmid (name mwr, 37,2)

$\mathrm{dd}=\operatorname{strmid}($ name mwr, 39,2)

hours $=$ float (strmid (name mwr, 42,2))

$\min =$ float $($ strmid (name_mwr $r, 44,2))$

$\mathrm{sec}=$ float (strmid (name mwr, 46,2$))$

shift $=3600 *$ hours $+60 *$ min + sec

$\mathrm{tl}=\mathrm{mm}+\mathrm{I}^{-}+\mathrm{dd}+\mathrm{I}^{-}$'+year

cdfid in mwr = ncdf open (name mwr, /NOWRITE)

if cdfid_in_mwr eq $\overline{-1}$ then message, 'cannot open file'

print, name_mwr

;

read array dimension

dimension id = ncdf dimid(cdfid in mwr, 'time')

if dimension id eq -1 then message, \$

' unab̄le to get dimension in mwr file'

ncdf_diming, cofid_in_mwr, dimension_id, time, size

read time_offset

time offset id = ncdf varid(cdfid in mwr, 'time offset')

if time_offiset_id eq $\overline{-1}$ then message, $\$$

' unable to get time offset in mwr file'

ncdf_varget, cdfid_in_mwr, time_offset_id, time_offset

time_offset ${ }^{-}=$time_offset $\overline{+}$ shift 
liq id = ncdf varid(cdfid in mwr, 'liq')

if $\overline{l i q}_{\text {id }}$ eq -1 then messäge, \$

'unable to get liq in mwr file'

$\operatorname{liq}=\operatorname{liq} * 10$ ncdf_varget, cofid_in_mwr, liq_id, liq

name_mpl $=$ './input $/ \mathrm{mpl} /$ '+month+'/'+nm_mpl (ind_mpl)

; in the winter month the mpl file name strings are longer

; by four character ("nor1")

if ( (mt ne 01) and (mt ne 02)) then begin

$h=0$

endif else begin

$\mathrm{h}=4$

endelse

hours = float (strmid (name $\mathrm{mpl}, 43+\mathrm{h}, 2)$ )

$\min =$ float $($ strmid $($ name $m p l, 45+h, 2))$

$\mathrm{sec}=$ float $($ strmid (name $m p l, 47+\mathrm{h}, 2)$ )

iprint, hours, min, sec

shift $=3600 *$ hours $+60 *$ mintsec

cdfid in mpl = ncdf open (name mpl, /NOWRITE)

if cdfid_in mpl eq -1 then message, 'cannot open file'

print, name mpl

read array dimension

dim_mpl_id $=$ ncdf_dimid(cdfid_in_mpl, 'time')

if $\overline{d i m}$ mpl id eq $\overline{-1}$ then messäge, \$

'unable to get dimension in mpl file'

ncdf diminq, cdfid_in mpl, dim mpl_id, time, size mpl

read time offset

time_offset_id_mpl $=$ ncdf_varid(cdfid_in_mpl, 'time_offset')

if time offset id mpl eq -1 then message, \$

' ' unable to $\bar{e}$ get time_offset in mpl file'

ncdf varget, cdfid in mpl, time offset id mpl, time offset mpl

time_offset_mpl $=$ time_offset_mpl+shift

;

read cloud_base_height

cbh_id = ncdf_varid(cdfid_in_mpl, 'cloud_base_height')

if $\overline{c b h}$ id eq $\overline{-1}$ then messāge, \$

'unable to get cbh in mpl file'

ncdf_varget, cdfid_in_mpl, cbh_id, cbh

;

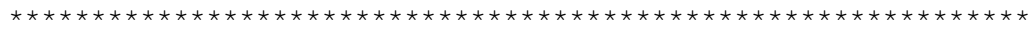

Set of thick marks (hours).

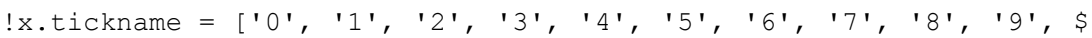

'10', '11', '12', '13', '14', '15', '16', '17', '18', '19', '20', \$

'21', '22', '23', '24']

!x.ticks $=24$

!x. style $=1$

;

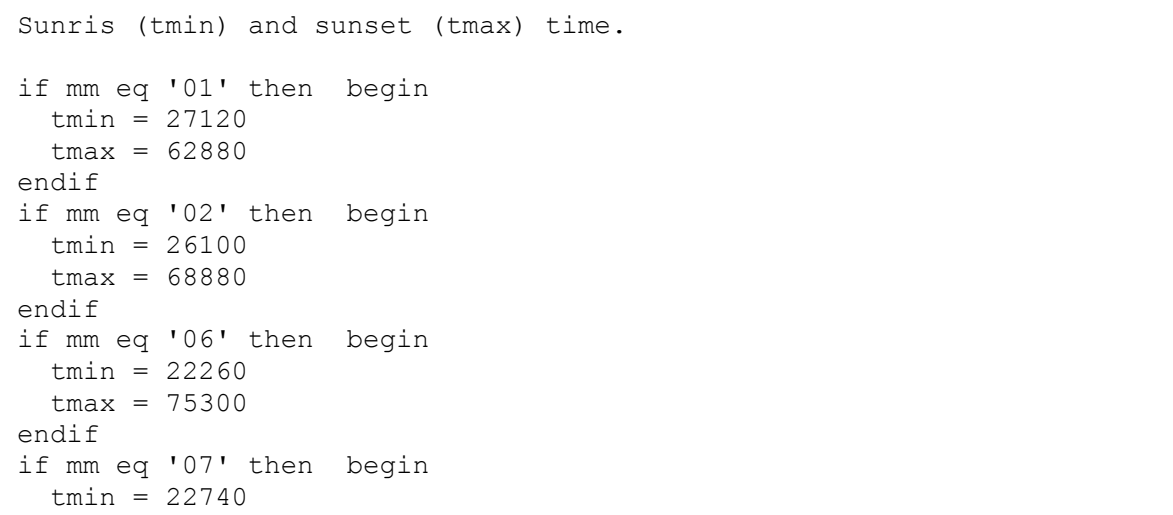




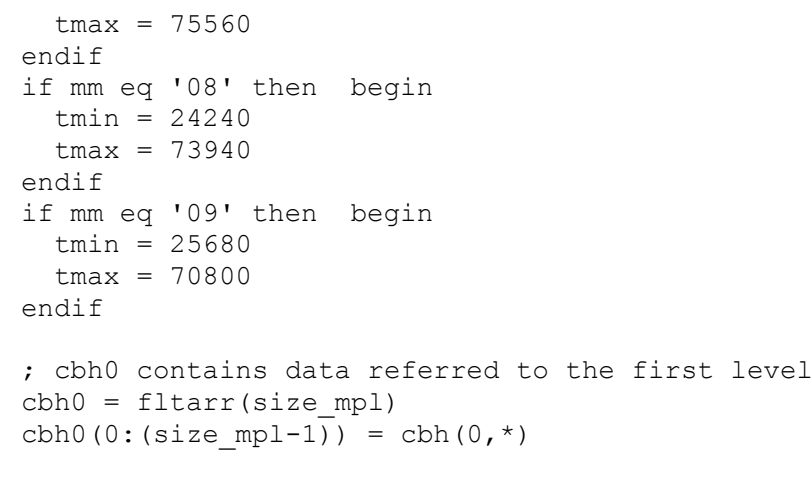

Axes and labels plot.

plot, /normal, /nodata, time offset, liq, xrange=[0.0,86400.0], \$ yrange $=[0,0.1]$, title $=t l$, xtitle $=$ "Time (hour)", $\$$ ytitle = "Total liquid water along LOS path $(\mathrm{mm}) ", \quad$ " xmargin $=[8,8]$, ymargin $=[4,4]$, color $=1$

; note: 200 is the factor that allow to obtain a $[0,20]$ range

; $\quad$ starting from a $[0,0.1]$ range.

axis, $86400,0,0$, yaxis $=1$, yrange $=! y \cdot$ crange $200, \$$ ystyle $=1, \$$ ytitle = "Cloud Base Height $(\mathrm{Km}) "$, color $=1$ 
oplot, time_offset, liq, color $=2$

Plot of the total cloud base height versus time.

; cbho/200 to obtain a properly scaled value of cloud base height

; with the actual scale for it

oplot, time_offset_mpl, cbho/200, color $=1$, psym $=1$, symsize $=0.5$

; $\quad$ Plot of mean value line (if mean is positive)

if mean gt 0 then begin

$x \times 3=[t m i n, t \max , t \max , t \min ]$

yy3 = [mean, mean, mean+0.002, mean+0.002]

endif

polyfill, $x x 3$, yy3, $\operatorname{color}=2$

Labels plot and positioning.

str1 = 'Mean: '+string (format $=$ ' (A10)', mean) +' (mm)'

str2 = 'Standard Dev.: '+string (format $=$ ' $(\mathrm{A} 10)$ ', $\mathrm{sd})+$ ' $(\mathrm{mm})$ '

xyouts, $3600,0.09$, color $=1, \$$

str1, charsize $=1.1, \$$

charthick $=1.5$

$; \mathrm{d}=(\max (1 i q)-\min (l i q)) / 10$

$\mathrm{d}=0.01$

xyouts, 3600, 0.09-d, str2, charsize $=1.1, \operatorname{col}$, $=1, \$$

charthick $=1.5$

Computation of mean and standard deviation for each hour.

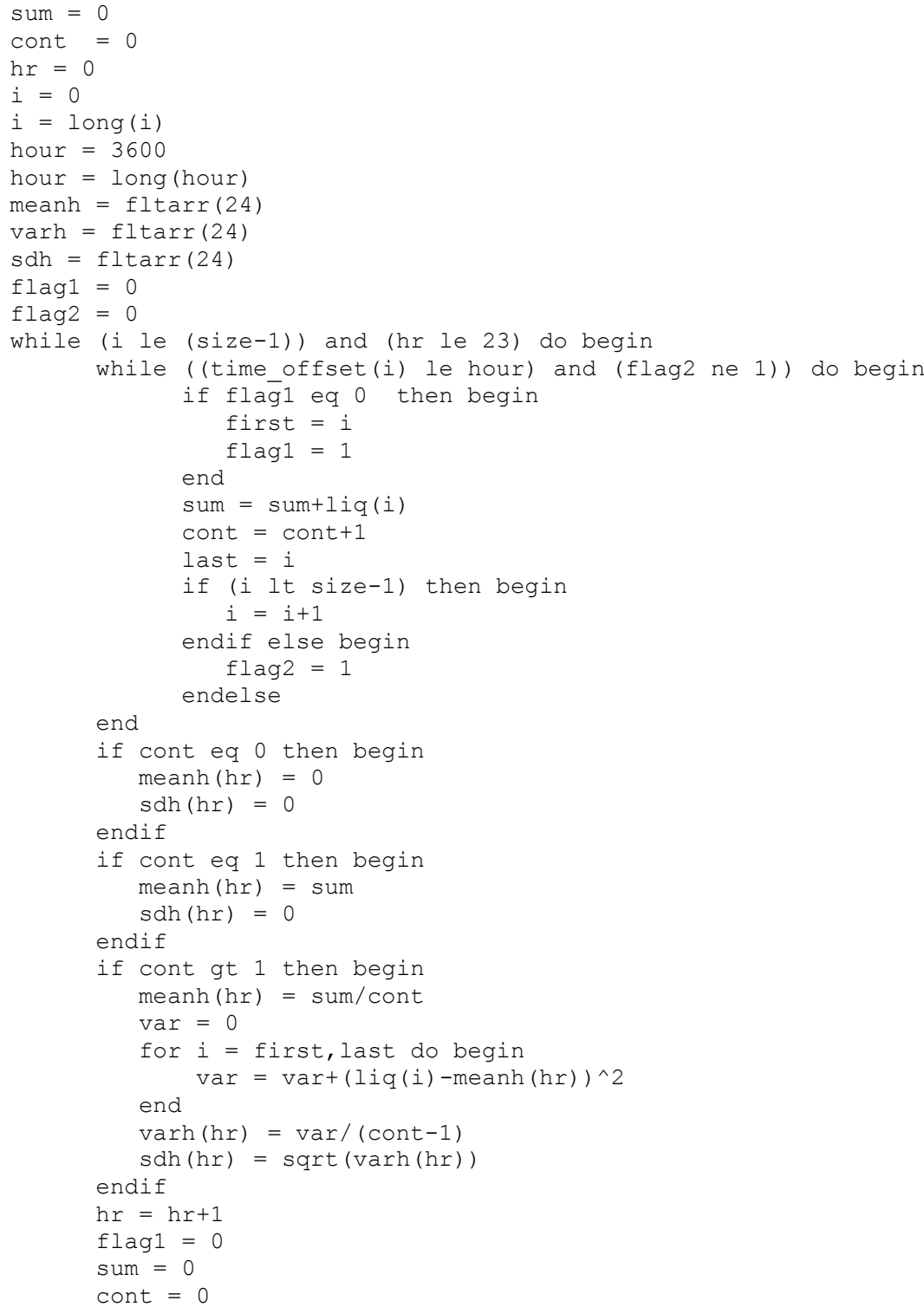


end

Second plot: table with the mean and standard deviation for each hour.

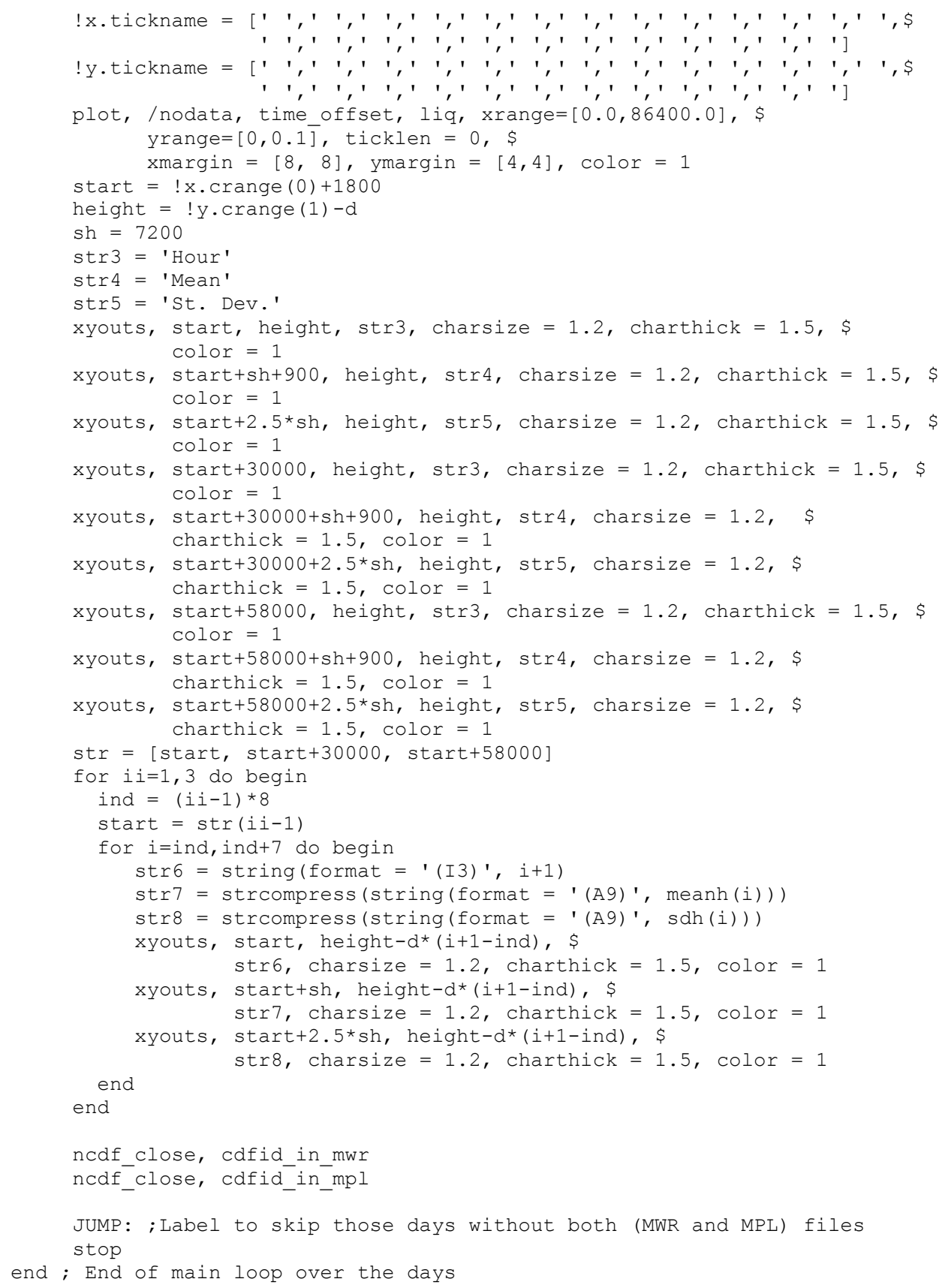

